\title{
AFM Height Measurements of Molecular Layers of a Carbocyanine Dye
}

\author{
Valery V. Prokhorov, Sergey I. Pozin, Dmitry A. Lypenko, Olga M. Perelygina, Eugene I. Mal’tsev, \\ Anatoly V. Vannikov \\ Frumkin Institute of Physical Chemistry and Electrochemistry, Russian Academy of \\ Sciences, Moscow, Russia \\ E-mail:vprokh@gmail.com
}

Received May 24, 2011; revised June 13, 2011; accepted June 23, 2011

\begin{abstract}
Atomic force microscopy (AFM) was used for the morphological characterization and precise height measurements of two-dimensional molecular layers of carbocyanine dye 3,3'-di( $\gamma$-sulfopropyl)-4,4',5,5'-dibenzo-9-ethylthiacarbocyanine betaine pyridinium salt. The AFM measurements reveal three morphological types of molecular aggregates: leaves, stripes and spots. The leaves are stripes have same monolayer height $\sim 1.4 \mathrm{~nm}$ and different crystal shapes: the leaves are monoloyers with the lens shape and the stripes are bilayers with the shape of extended rectangles. The monolayer height $\sim 1.4 \mathrm{~nm}$ was interpreted as indicating the symmetrical packing arrangement of dye molecules. In the symmetrical monolayer, the sulfopropyl groups of all-trans monomer units are located on both monolayer sides whereas the adjacent stacked dye molecules have a lateral slippage providing the J-aggregate optical properties. The lower height of spots $\sim 1 \mathrm{~nm}$ was explained by the model of an asymmetric monolayer with sulfopropyl groups of all-trans monomers occupying the same position with respect to the monolayer plane. The packing arrangement of all-trans monomers in the asymmetric monolayer corresponds to $\mathrm{H}$-aggregate. The alternative models of the packing arrangement in monolayers with mono-cis1 monomer configuration are discussed.
\end{abstract}

Keywords: Atomic Force Microscopy, J-Aggregates, H-Aggregates, Monolayers

\section{Introduction}

Cyanine dyes have a large conjugated $\pi$-system and easily self-assemble into different kinds of supramolecular structures (aggregates). The aggregates are classified into two general classes referred to as J- and H-aggregates, characterized respectively by red or blue shifts of their intense absorption spectra with respect to that of monomers [1]. Their unusual optical properties result from the formation of delocalized Frenkel-type exciton states [2] in the stacked adjacent interacting chromophores. The molecular transition dipoles in aggregates are parallel to each other and shifted in adjacent molecular rows by a slip angle $\alpha$ (the angle between the centers of mass of two adjacent dye molecules in adjacent rows and the long axes of these molecules) [2]. The slip angle determines the sign of the spectral absorption shift: the large molecular slippage (e.g. $\alpha<32^{\circ}$ ) results in a red shift (in J-aggregates), while the small slippage $\left(\alpha>32^{\circ}\right)$ results in a blue shift (in H-aggregates). Generally the dye samples demonstrate complex morphological and structural variability so that different structures can be found in different solutions and at different dye concentrations. For thiacyanine (TC) and some carbocyanine dyes the transmission electron microscopy (TEM) and atomic force microscopy (AFM) demonstrate several morphological groups of J-aggregates: extended two-dimensional rods and stripes [3], quasi one-dimensional stripes, twisted ribbons and tubules [4,5]. The structural and morphological complexity was found for 3,3'-disulfopropyl-5,5'-dichloro-9-methyl thiacarbocyanine (TCC). It was shown that $\mathrm{J}$-aggregates and $\mathrm{H}$-aggregates could be found independently or coexist in dependence on the dye concentration or the counteraction type in water solutions $[6,7]$.

It was shown that J-aggregates nanocrystalline particles can be used as dopants to electroactive polymers for the construction of nanoscale materials with useful opto- 
electronic properties such as efficient tunable photoluminescence and electroluminescence, ultrafast optical switching, electron-hole conductivity [8,9]. Typically, a building unit of the dye molecular aggregates is a monolayer (or bilayer) formed by stacked dye molecules with molecular planes oriented normally to the monolayer plane. The measurements of the monolayer(s) thickness thus give important structural information on a type of molecular packing. The bilayer thickness of some carbocyanine dyes was determined by TEM from the visualization of tubular walls or the stripe patterns of twisted ribbon stacks taken in "edge-on" orientation [4]. The obtained results indicated the bilayer structure in which two asymmetric monolayers partially interpenetrate. The advantage of AFM in comparison with TEM is the capability for direct measurements of heights of mono- or multilayers with potentially much higher precision of the order of tenth of angstrom. High-resolution AFM measurements were conducted for J-aggregates of two dyes [10] and the AFM heights of their monolayers were precisely determined from the mono- and multilayers height distributions. In contrast to AFM results for the TC dye reported in [3], the smaller monolayer height $\sim 1.0-1.1 \mathrm{~nm}$ was found, indicating the asymmetric monolayer structure with sulfopropyl groups on one side of the monolayer. The AFM heights derived in [10] for the narrow stripes of the C8S3 carbocyanin dye characterized by TEM in [4] were strictly quantized with a step $\sim 3$ $\mathrm{nm}$. This height step corresponds to the bilayer with partially interpenetrated long aliphatic chains belonging to individual monolayers.

In the present work, a carbocyanine dye with larger heterocyclic system was chosen with the aim of AFM morphological characterization and the precise height measurements of the constituent molecular layers.

\section{Experimental Details}

The chemical structure of the carbocyanine dye used (refereed below as D-1) 3,3'-di( $\gamma$-sulfopropyl)-4,4',5,5'dibenzo-9-ethylthiacarbocyanine betaine pyridinium salt is represented in Figure 1. In comparison with the cya

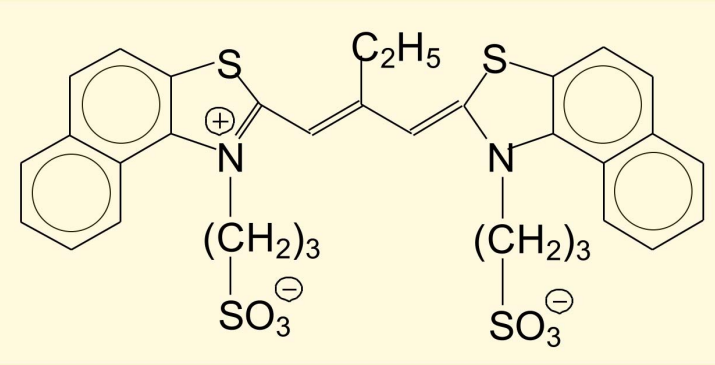

Figure 1. The chemical structure of the dye D-1. nine dyes mentioned above it has larger $\pi-\pi$ conjugated system. Molecular aggregates were deposited on a freshly cleaved mica surface at room temperature for a time $3 \mathrm{~min}$ from water solutions with concentration of $\sim 0.5 \mathrm{mg} / \mathrm{ml}$. After the deposition the solution excess was blown out by the air stream.

AFM measurements were conducted using Solver-Bio (NT-MDT, Zelenograd, Russia) operating at ambient conditions. The standard silicon cantilevers (Olympus, Japan) with the spring constant $\sim 40 \mathrm{~N} / \mathrm{m}$ and curvature radius $\sim 10$ $\mathrm{nm}$ were used. The attraction regime of probe-surface interactions with small amplitudes of probe oscillations (typically $\sim 10 \mathrm{~nm}$ ) was used. It provided non-contact "soft" scanning without surface deformation by an AFM probe in contrast to the repulsion regime in which the probe directly touches the sample and can irreversibly damage it [11]. The routine off-line AFM image analysis was performed with the use of the software Femtoscan (http://spm.genebee.msu.ru/products/ fmsprog.html).

\section{Results and Discussion}

Figure 2(a) represents the AFM topography image of two- dimensional sheets of D-1 molecular aggregates. Three morphologically different structures are observed with characteristic difference in a shape and a height, correspondingly the leaves $(\mathrm{A})$, the stripes $(\mathrm{B})$ and the spots (C). The elongated leaves have curved shapes in contrast with the extended stripes with parallel long sides, whereas the spots have round irregular shape. The height sections s1-s3 (Figure 2(b)) pass through the structures of these three types. It is seen that the height of the stripes $\sim 2.6-$ $2.8 \mathrm{~nm}$ is twice that of the leaves $(\sim 1.3 \mathrm{~nm})$, whereas the spots have lowest height $\sim 0.9 \mathrm{~nm}$. The overlapping of two leaves in the upper left part of Figure 2(a) indicates unambiguously that they have been originally formed in the solution bulk and overlapped while the adsorption. The leaves formation due to a surface-induced mechanism [10] can thus be excluded. The profile b1 indicates that the height of an overlapped area is exactly twice the leaves height. The overlapping was observed for the stripes as well (data not represented). Contrary to the leaves and the stripes, the overlapping of the spots was not observed indicating probably the surface growth mechanism of spots formation.

It is worth comparing the structures shown in Figure 2(a) with those already reported in literature for some other dyes. One can see very close morphological similarity revealed by AFM for the stripes in Figure 2(a) and for bilayer rod-like TC J-aggregates in [3]. The steps (marked by the double asterisk in Figure 2(a)) formed by two mono- layers are observed upon open ends of some stripes similar to those of reported for TC rods in [3]. It is notable that the opposite end of the stripes in Figure 2(a) is rectangular (marked 

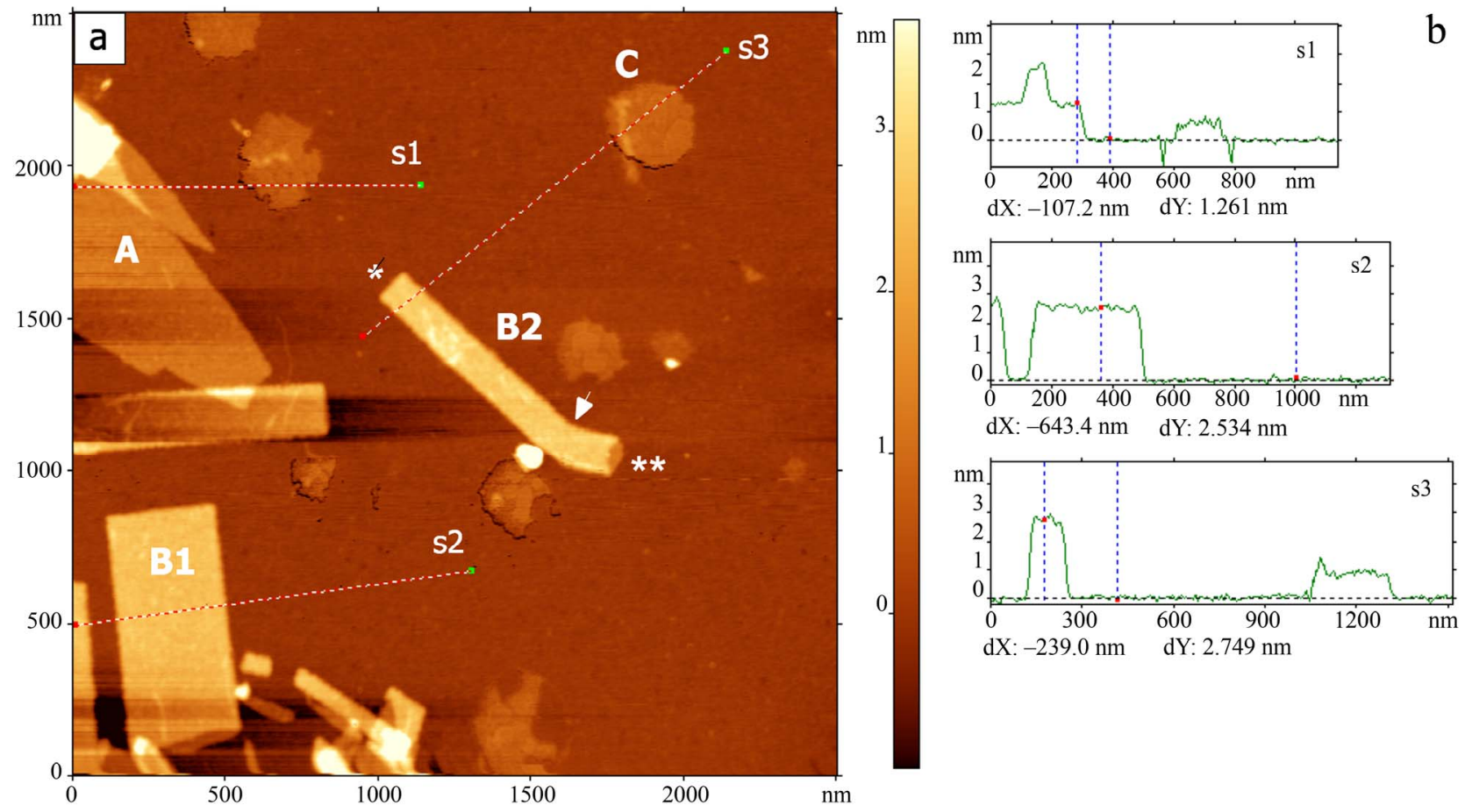

Figure 2. (a) The AFM height image of molecular aggregates of the dye D-1: A-monolayer leaves, B-bilayer stripes, $\mathrm{C}$-monolayer spots. The asterisk and double asterisk show respectively the straight rectangular and the ragged open ends of the stripe B2, the arrow shows the salient point of the stripe B2. Scan size $2500 \mathrm{~nm}$. (b) The height profiles through the white dashed lines correspondingly s1 to $s 3$ in Figure 2(a).

by an asterisk). The AFM height of the stripes ( 2.6 - 2.8 $\mathrm{nm})$ is close to that of observed for TC rods $(\sim 3 \mathrm{~nm})$ as reported in [3]. The bilayer rod morphology was interpreted in [3] as corresponding to tubular morphology in a solution phase squeezed by interaction with a substrate at adsorption. Our results bring this interpretation into challenge. Particularly, the narrow stripe B2 in Figure 2(a) has a convex point (shown by the arrow) with no visible rapture that could appear at surface adsorption, which is difficult to explain in terms of the tubular model. Moreover, physically conceivable tubular models with walls comprised of a single monolayer must very probably imply a constant tubular diameter (and thus the width of stripes). The diameter constancy is dictated by a set of relevant physical parameters influencing the tube curvature, in particular by mono- layer flexibility. This expectation contradicts the experiment, i.e. the width of stripes in Figure 2(a) such as B1 and B2 is not fixed and varies in a wide range (see also the narrowest short stripes in the bottom of Figure 2(a)).

Another dye (MTC) with very similar structure $\left(\mathrm{CH}_{3}\right.$ group instead of $\mathrm{C}_{2} \mathrm{H}_{5}$ group in the spacer) formed $\mathrm{H}$-aggregates with a linear fibrillar morphology in interfacial assemblies with gemini amphiphiles. [12] Interestingly, the $\mathrm{H}$ - to J-transition was observed in such as- semblies upon exposure to $\mathrm{HCl}$ gas, and the thus-formed J-aggregates could be reversibly changed into H-aggregates under a hot water vapor. The coexistence of both $\mathrm{J}$ - and $\mathrm{H}$-aggregates and their transformation with time was also registered for D-1 in binary water-ethanol mixtures [13]. The absorption spectrum of the dye contains optical bands of the mono-cis and trans forms, as well as the aggregated states (dimer, H-aggregate, and J-aggregate). A cis-trans isomer equilibrium was found to shift toward the cis-isomer at room temperature. The rate of formation of J-aggregates correlates with the rate of decay of dimers or monomers and is dependent on the type of metal ion and temperature [14]. The absorption spectra of binary random molecular mixtures of D-1 with very similar thiacarbocyanine dye have been studied with the aim of modeling the optical absorption of two-dimensional mixed molecular aggregates [15]. The optical spectroscopy and wide angle X-ray scattering were used in characterization of D-1 aggregates incorporated into a Na-polystyrene-sulfonate matrix [16]. These results revealed the formation of J-aggregates, however direct microscopic visualization was not provided.

Thus one may conclude that the morphological picture is very mosaic for this dye. It depends on many factors, such as counter ions and dye concentration. It is evident 
that structural correspondence for the morphologies observed in Figure 2(a) can't be done unambiguously on the basis of only AFM data and below we consider several alternative ways of structural interpretation. Heights of the leaves and stripes observed by AFM (Figure 2(b)) agree well with the model of a symmetric monolayer made of monomers in all-trans conformation (Figure 3(b)). In this model, the long axes of dye molecules are oriented parallel to each other and to mica plain whereas the molecular planes are oriented perpendicular to mica ("edge-on" orientation). To satisfy the AFM height data, the model implies that adjacent stacked molecules have anti-parallel orientation in the up-down direction so that aliphatic chains occupy both sides of the monolayer. The anti-parallel up-down arrangement follows from the fact that monolayer height observed by AFM substantially exceeds the upper border for a vertical molecular dimension which is estimated to be $\sim 1.05 \mathrm{~nm}$ (Figure 3(a)). For the anti-parallel up-down orientation the requirement of the efficient overlapping of $\pi$-electron chromophore systems is achieved at the lateral translation (slippage) of two adjacent stacked molecules by $\sim 1.3 \mathrm{~nm}$ (see Figure 3(b)). This translation produces the small slip angle $(\alpha)$ corresponding to the J-aggregate dimer: $\alpha=\operatorname{atan}(0.4$ $\mathrm{nm} / 1.3 \mathrm{~nm}) \sim 17 \mathrm{deg}$. At the estimation of the slip angle, the plane-to-plane intermolecular distance was assumed to be equal $\sim 0.4 \mathrm{~nm}[15,17]$. The represented in Figure 3(b) symmetric monolayer height $\sim 1.4 \mathrm{~nm}$ is the upper boundary corresponding to the monomer configuration with fully extended aliphatic chains. For tilted chains, the height is smaller. The leaves are assumed to be single symmetric monolayers whereas the stripes consist of two such monolayers. The above consideration implies a small slip angle, thus both the leaves and the stripes are assumed to have the J-aggregates optical properties. The observed rectangular shapes of stripes could be explained by the J-aggregate growth of the ladder type (such as represented in Figure 3(b)) having intrinsically rectangular geometry in contrast with the alternative inclined staircase packing arrangement in J-aggregates.

It is noteworthy to say that the molecular modeling for this dye conducted in [16] reveals that the alternative configuration of the monomer unit, i.e. the mono-cis1 (instead of all-trans configuration implied in the models in Figure 3) proved to be energetically advantageous. It was shown that the mono-cis configuration provides three new types of the packing arrangement in J-aggregates, i.e. one linear planar and two helical packing arrangements. The helical packing arrangements with a diameter within $1.2-2.0 \mathrm{~nm}$ seem to be excluded from our consideration because they generate intrinsically linear fibrillar structures. However, at present it is not clear whether the remained model of the linear mono- cis1 packing arrangement in [16] can be applied for the description of the structures and their heights observed in Figure 2(a).

The dramatic morphological difference between the leaf morphology (with a height of a symmetric monolayer) and the stripe morphology (with a double monolayer height) should be noted and needs a special consideration. Its explanation can be performed in two basically different ways. First, it may be supposed that both morphologies correspond to the same J-aggregate crystal structure with the same monomer configuration and the same slip angle (supposedly in accordance with the model in Figure 3(b)). However for the leaf-like monolayer and the stripe-like bilayer, the growth of the crystal sheets proceeds along different crystallographic directions. The difference in crystallographic growth directions generates the difference in the geometrical shapes and observed peculiarities at a macroscopic scale (such as straight linear or curved crystal faces). The difference in geometrical shapes is in this case due to peculiarities of crystal growth for the same crystal structure. Second, it may be supposed that the leaves and stripes have different crystal structures, i.e. the elementary cells and/or monomer conformations.

For the spots, the monolayer height $\sim 0.9 \mathrm{~nm}$ is noticeably lesser than that of $\sim 1.4 \mathrm{~nm}$ for the leaves. The lower monolayer height can be explained by two different ways. First, it is explained by the model in Figure 3(c) with all-trans monomer configuration and parallel orientation of adjacent stacked molecules. To satisfy the AFM results, the entire sulfopropyl group must occupy the same position with respect to the monolayer plane. Thus Figure 3(c) represents a model of the asymmetric monolayer. Evidently, the best overlapping of $\pi$-electrons at chromophores stacking is achieved in this case for the slip angle $=90 \mathrm{deg}$, resulting in the $\mathrm{H}$-aggregate optical spectra. An alternative explanation can be probably provided in accordance with the model in [16], i.e. by the stacking of monomers arranged in mono-cis 1 configuration. Both the mono-cis1 molecular configurations in [16] and the all-trans configuration considered above in Figure 3(c) have close vertical geometrical dimensions $\sim 1 \mathrm{~nm}$. The particular details of probable mono-cis1 monomers stacking in the monolayer with a height $\sim 1 \mathrm{~nm}$ in accordance with the alternative model developed in [16] remain however unclear and need to be modeled more accurately.

The results considered above demonstrate the morphological and structural complexity of the dye molecular aggregates at a meso-scale. Complementary information on the anisotropy of molecular sheets could be indispensable for making unambiguous conclusions on the structure of leaves, stripes and spots. The polarized opti- 
(a)

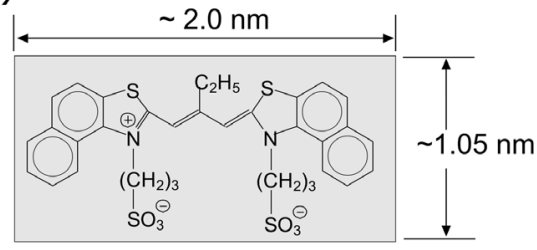

(b)

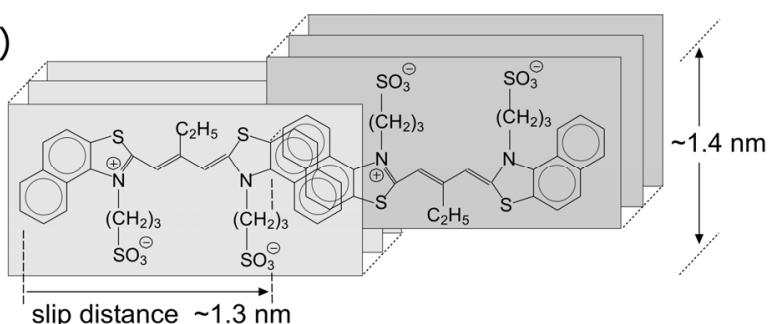

(c)

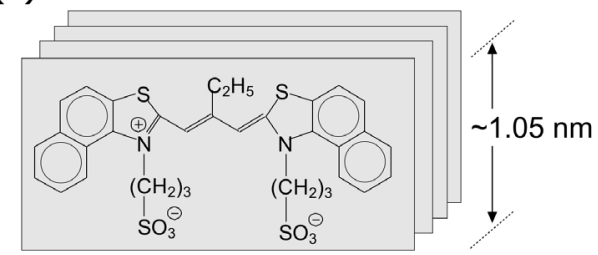

Figure 3. (a) The schematic representation of D-1 molecule with lateral dimensions of $2.0 * 1.05 \mathrm{~nm}$. (b,c) The models of two expected energetically favorable dispositions of adjacent stacked dye molecules in all-trans configuration in the aggregate monolayer: (b) J-aggregate of a ladder type with a monolayer height $\sim 1.4 \mathrm{~nm}$, (c) $\mathrm{H}$-aggregate with a height $\sim 1.05 \mathrm{~nm}$.

cal microscopy (POM) measurements have a limited spatial resolution so that the distinguishing of observed by AFM structures such as in Figure 2(a) is problematic for POM. More certain results could be obtained by scanning near-filed optical microscopy (SNOM) measurements similar to those of conducted for TC in [3]. In comparison with optical microscopy, the SNOM technique has intrinsically larger lateral resolution, thus providing information on both the aggregates shapes in the submicron range and their optical properties (i.e. fluorescence, linear dichroism etc.). These measurements are planned in the future.

\section{Conclusions}

AFM indicates variability in both a morphology (leaves, stripes, spots) and a height of D-1 molecular layers ( $\sim 1.3$ - $1.4 \mathrm{~nm}$ and $\sim 0.9 \mathrm{~nm}$ ). Interestingly, the leaves and stripes have essentially different crystallographic shapes but their monolayer height is same $\sim 1.3-1.4 \mathrm{~nm}$. The spots height is $\sim 0.9 \mathrm{~nm}$. The heights $1.4 \mathrm{~nm}$ and $1.0 \mathrm{~nm}$ are explained well by the models of correspondingly symmetrical and asymmetrical monolayers with all-trans configuration of dye monomers. In the symmetrical (asymmetrical) monolayer the sulfopropyl groups of all-trans monomer units are located on both (single) monolayer side(s). The symmetric monolayers (leaves and stripes) are very probably $\mathrm{J}$-aggregates, whereas the asymmetric monolayers (spots) are H-aggregates.

\section{Acknowledgements}

We thank Professor Boris I. Shapiro (Photographic Chemistry Institute, Moscow), for kindly providing the thiacarbocyanine dye.

\section{References}

[1] F. C. Spano, "The Spectral Signatures of Frenkel Polarons in H- and J-Aggregates," Accounts of Chemical Research, Vol. 43, No. 3, 2010, pp. 429-439. doi:10.1021/ar900233v

[2] V. Czikkely, H. D. Försterling and H. Kuhn, "Extended Dipole Model for Aggregates of Dye Molecules," Chemical Physics Letters, Vol. 6, No. 3, 1970, pp. 207-210. doi:10.1016/0009-2614(70)80220-2

[3] H. Yao, "Morphology Transformations in Solutions: Dynamic Supramolecular Aggregates," Annual Repports on the Progress of Chemistry, Section C, Vol. 100, 2004, pp. 99-148. doi:10.1039/b313661m

[4] R. H. von Berlepsch, C. Böttcher, A. Ouart, C. Burger, S. Dähne and S. Kirstein, "Supramolecular Structures of JAggregates of Carbocyanine Dyes in Solution," The Journal of Physical Chemistry B, Vol. 104, No. 22, 2000, pp. 5255-5262. doi:10.1021/jp000220z

[5] H. von Berlepsch, S. Kirstein and C. Böttcher, "Supramolecular Structure of J-Aggregates of a Sulfonate Substituted Amphiphilic Carbocyanine Dye in Solution: Methanol-Induced Ribbon-to-Tubule Transformation," The Journal of Physical Chemistry B, Vol. 108, No. 48, 2004, pp. 18725-18733. doi:10.1021/jp046546f

[6] H. Yao, K. Domoto, T. Isohashi and K. Kimura, "In Situ Detection of Birefringent Mesoscopic H- and J-Aggregates of Thiacarbocyanine Dye in Solution," Langmuir, Vol. 21, No. 3, 2005, pp. 1067-1073. doi:10.1021/la0479004

[7] H. Yao, T. Isohashi and K. Kimura, "Electrolyte-Induced Mesoscopic Aggregation of Thiacarbocyanine Dye in Aqueous Solution: Counterion Size Specificity," The Journal of Physical Chemistry B, Vol. 111, No. 25, 2007, pp. 71767183. doi:10.1021/jp070520h

[8] E. I. Mal'tsev, D. A. Lypenko, B. I. Shapiro, M. A. Brusentseva, G. H. W. Milburn, J. Wright, A. Hendriksen, V. I. Berendyaev, B. V. Kotov and A. V. Vannikov, "Electroluminescence of Polymer/J-Aggregate Composites," Applied Physics Letters, Vol. 75, No. 13, 1999, pp. 18961898.

[9] E. I. Mal'tsev, D. A. Lypenko, V. V. Bobinkin, A. R. Tameev, B. I. Shapiro, H. F. M. Schoo and A. V. Van- 
nikov, "Near-Infrared Electroluminescence in Polymer Composites Based on Organic Nanocrystals," Applied Physics Letters, Vol. 81, No. 16, 2002, pp. 3088-3090.

[10] V. V. Prokhorov, E. I. Mal'tsev, O. M. Perelygina, D. A. Lypenko, S. I. Pozin and A. V. Vannikov, "High Precision Nanoscale AFM Height Measurements of J-Aggregates," Nanotechnology in Russia, Vol. 6, No. 5-6, 2011, pp. 286-297. doi:10.1134/S199507801103013X

[11] R. Garcia and R. Pérez, "Dynamic Atomic Force Microscopy Methods," Surface Science Reports, Vol. 47, No. 6-8, 2002, pp. 197-301. doi:10.1016/S0167-5729(02)00077-8

[12] G. Zhang and M. Liu, "Interfacial Assemblies of Cyanine Dyes and Gemini Amphiphiles with Rigid Spacers: Regulation and Interconversion of the Aggregates," The Journal of Physical Chemistry B, Vol. 112, No. 25, 2008, pp. 7430-7437. doi:10.1021/jp8005298

[13] M. V. Alfimov, A. A. Shtykova and V. F. Razumov, "Photo- and Thermoinitiated Formation of J- and H-Aggregates in Amorphous Dispersion of a Carbocyanine Dye," High Energy Chemistry, Vol. 40, No. 1, 2006, pp.
18-21.

[14] T. D. Slavnova, A. K. Chibisov and H. Görner, "Kinetics of Salt-Induced J-Aggregation of Cyanine Dyes," The Journal of Physical Chemistry A, Vol. 109, No. 21, 2005, pp. 4758-4765. doi:10.1021/jp058014k

[15] L. D. Bakalis, I. Rubtsov and J. Knoester, "Absorption Spectra of Mixed Two-Dimensional Cyanine Aggregates on Silver Halide Substrates," The Journal of Chemical Physics, Vol. 117, No. 11, 2002, pp. 5393-5403. doi: $10.1063 / 1.1499958$

[16] G. Busse, B. Frederichs, N. Kh. Petrov and S. Techert, "Structure Determination of Thiacyanine Dye J-Aggregates in Thin Films: Comparison between Spectroscopy and Wide Angle X-Ray Scattering," Physical Chemistry Chemical Physics, Vol. 6, 2004, pp. 3309-3314. doi:10.1039/b400212a

[17] G. N. Chuev and M. V. Fedorov, "Reference Interaction Site Model Study of Self-Aggregating Cyanine Dyes," The Journal of Chemical Physics, Vol. 131, No. 7, 2009, Article ID: 074503. 